\title{
Ubiquitin-fold modifier 1 acts as a positive regulator of breast cancer
}

\section{Hee Min Yoo, Jong Ho Park, Young Joo Jeon and Chin Ha Chung*}

Institute for Protein Metabolism, School of Biological Sciences, Seoul National University, Seoul, South Korea

Edited by:

Wen Zhou, Columbia University, USA

Reviewed by:

Guan Chen, Medical College of

Wisconsin, USA

Weiyi Toy, Memorial Sloan Kettering

Cancer Center, USA

*Correspondence:

Chin Ha Chung, Institute for Protein Metabolism, School of Biological

Sciences, Seoul National University,

56-1 Shillim-dong, Gwanak-gu, Seoul

151-742, South Korea

e-mail: chchung@snu.ac.kr
Estrogen receptor- $\alpha(E R \alpha)$ is a steroid hormone-sensitive transcription factor that plays a critical role in development of breast cancer. The binding of estrogen to $E R \alpha$ triggers the recruitment of transcriptional co-activators as well as chromatin remodeling factors to estrogen-responsive elements ( $E R E)$ of $E R \alpha$ target genes. This process is tightly associated with post-translational modifications (PTMs) of ER $\alpha$ and its co-activators for promotion of transcriptional activation, which leads to proliferation of a large subset of breast tumor cells. These PTMs include phosphorylation, acetylation, methylation, and conjugation by ubiquitin and ubiquitin-like proteins. Ubiquitin-fold modifier 1 (UFM1), one of ubiquitin-like proteins, has recently been shown to be ligated to activating signal co-integrator 1 (ASC1), which acts as a transcriptional co-activator of nuclear receptors. Here, we discuss the mechanistic connection between ASC1 modification by UFM1 and ER $\alpha$ transactivation, and highlight how the interplay of these processes is involved in development of breast cancer. We also discuss potential use of UFM1-conjugating system as therapeutic targets against not only breast cancer but also other nuclear receptor-mediated cancers.

Keywords: ASC1, breast cancer, ER $\alpha$, post-translational modification, UFM1

\section{INTRODUCTION}

Protein modifications by ubiquitin and ubiquitin-like proteins, including SUMO and ISG15, have emerged as critical regulatory processes, such as in the control of cell cycle, stress response, signaling transduction, and immune response. Moreover, deregulation of the modification systems often gives rise to numerous human diseases, such as cancers, neurodegenerative diseases, and immune diseases (1-4).

Ubiquitin-fold modifier 1 (UFM1) is the most recently identified ubiquitin-like protein (5). Like ubiquitination, protein modification by UFM1 (ufmylation) utilizes a cascade three-enzyme system: UBA5 as an UFM1-activating E1 enzyme, UFC1 as an UFM1-conjugating E2 enzyme, and UFL1 as an UFM1 E3 ligase. This ufmylation process can be reversed by UFM1-specific proteases (UFSPs) (6). All of the proteins involved in reversible protein modification by UFM1 are conserved in metazoa and plants, but not in yeast, implicating its specific roles in multicellular organisms.

Abbreviations: AR, androgen receptor; ASC1, activating signal co-integrator 1; Cdk5rap3, CDK5 regulatory subunit-associated protein 3; DDRGK1, DDRGK domain-containing protein $1, E R \alpha$, estrogen receptor- $\alpha$; ISG15, interferonstimulated gene 15; LZAP, LXXLL/leucine zipper-containing alternative reading frame (ARF)-binding protein; Maxer, multiple $\alpha$-helix protein located at ER; NCAM, neuronal cell adhesion molecule; NLBP, novel LZAP-binding protein; RAR $\alpha$, retinoic acid receptor $\alpha$ RCAD, regulator of C53/LZAP and DDRGK1; SRC1, steroid receptor coactivator 1; SUMO, small ubiquitin-related modifier; TRIP4, thyroid hormone receptor interactor 4; UBA, ubiquitin-like modifier activating enzyme; UBE1DC1, ubiquitin activating enzyme E1-domain containing 1; UFBP1, UFM1-binding protein 1 containing a PCI domain; UFC1, ubiquitin-fold modifierconjugating enzyme 1; UFL1, UFM1-specific ligase 1; UFM1, ubiquitin-fold modifier 1; UFSP, UFM1-specific protease; Urm1, ubiquitin-related modifier 1.
Not only estrogen receptor $\alpha(\mathrm{ER} \alpha)$ itself but also its coregulators, including SRC1 and p300, are known to undergo a wide variety of post-translational modifications (PTMs), such as phosphorylation, acetylation, methylation, ubiquitination, and sumoylation. Moreover, these PTMs have been identified as critical events that regulate the expression of ER $\alpha$ and its transcriptional co-regulators and their stability, subcellular localization, and sensitivity to hormonal response (7-12). Although the components of estrogen signaling pathways are suitable and efficient targets for breast cancer therapies, the role of their PTMs in initiation and progression of breast carcinogenesis remains largely elusive.

Activating signal co-integrator 1 (ASC1), originally identified as thyroid hormone receptor interactor 4 (TRIP4), is one of ER $\alpha$ transcriptional co-activators (13). It also serves as a co-activator of other nuclear receptors, such as thyroid hormone receptor (TR) and retinoic acid receptor $\alpha($ RAR $\alpha)$ (14-16). However, it remained unknown whether ASC1 also undergoes PTMs and how the PTMs of ASC1 influence its co-activator function toward nuclear receptors.

In this review, we will provide an overview on current and emerging roles of the UFM1 system, with a focus on ASC1 ufmylation in regulation of breast cancer development. A thorough understanding of ASC1 ufmylation would promote not only the identification of new markers for prognosis of breast cancer but also the development of novel therapeutic strategies.

\section{PROPERTIES OF UFM1}

UFM1 consists of 85 amino acids with a predicted molecular mass of $9.1 \mathrm{kDa}$. Its gene is located in human chromosome 13q13.3. UFM1 is expressed in human cells as a precursor with a C-terminal Ser-Cys dipeptide extension, which needs to be processed by UFSPs 


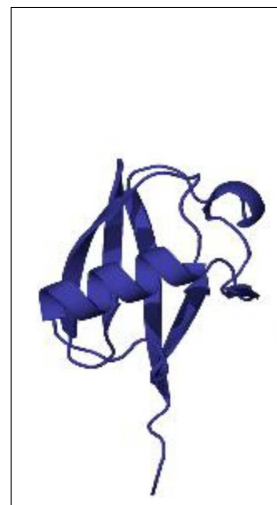

Ubiquitin

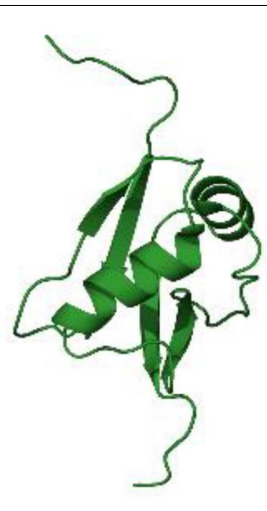

UFM1

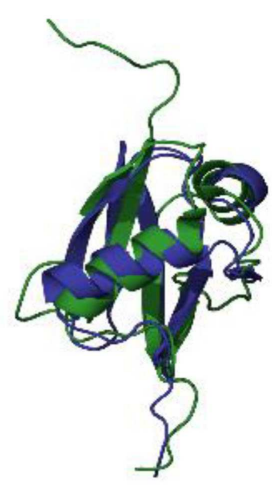

Merge
FIGURE 1 | 3D structures of ubiquitin and UFM1 in human. PDB IDs for ubiquitin and UFM1 are 1 UBO and 1 WXS, respectively.

prior to conjugation to target proteins (5). Matured UFM1 has a single glycine residue at the C-terminus, which also is required for conjugation to its target proteins, unlike ubiquitin and most other ubiquitin-like proteins, such as SUMO and NEDD8, which have a conserved C-terminal di-glycine. UFM1 is localized in both the nucleus and the cytoplasm (5).

Although UFM1 has a limited amino acid sequence identity $(\sim 16 \%)$ with ubiquitin, it displays a striking similarity in its tertiary structure to ubiquitin (Figure 1). UFM1 adopts an ubiquitin-like $\alpha+\beta$ fold with ordered $\beta-\beta-\alpha-\beta-\beta-\alpha-\beta$ secondary structure along the sequence (17). A special feature in UFM1 structure is the absence of the cluster of the acidic residues in the $\alpha 1$ surface, which is displayed by ubiquitin (17). Therefore, it has been suggested that UFM1 employs the uncharged surface for binding to its putative partners.

\section{ENZYMES FOR UFM1 MODIFICATION \\ UBA5}

UBA5 (also known as UBE1DC1), an UFM1-activating E1 enzyme, consists of 404 amino acids with a predicted molecular mass of $44.7 \mathrm{kDa}(18)$. Its gene is located in human chromosome 3q22.1. UBA5 is expressed in human as two distinct isoforms (amino acid sequences: 1-404 and 57-404) due to alternative splicing of its primary transcript $(19,20)$. The role of the additional N-terminal region (1-56) is unknown, as it is not required for UFM1 activation (19). Typically, E1 enzymes consist of the first and second catalytic cysteine half-domains (FCCH and $\mathrm{SCCH}$, respectively), the adenylation domain, and the C-terminal ubiquitin-fold domain (19). UBA5 lacks the FCCH and SCCH domains, but instead simply comprises an adenylation domain, in which the catalytic cysteine (Cys250) is located, and an ubiquitinfold domain $(19,20)$. Therefore, UBA5 is much smaller than other E1 enzymes, which comprise $>1,000$ amino acid residues. UBA5 is predominantly localized in the cytoplasm (20).

At the expense of ATP, UBA5 activates UFM1 (i.e., generates adenylated UFM1 and inorganic pyrophosphate). UFM1 is then conjugated to Cys 250 of UBA5 via a thioester bond with the release of AMP (5). It has been reported that UBA5 can also activate
SUMO2 under both in vitro and in vivo conditions (20). However, the loss of mouse UBA5 has no effect on the conjugation of ubiquitin-like proteins to cellular proteins, except that of UFM1 (21). In addition, overexpression of UBA5 promotes the modification of ASC1 by UFM1, but not by any other ubiquitin-like proteins (22), indicating that UBA5 is a specific E1 enzyme for UFM1.

Significantly, UBA5-deficient mice die in utero due to severe anemia associated with defective differentiation of both megakaryocytes and erythrocytes, although UBA5 is dispensable for the production of erythropoietin (21). Moreover, transgenic expression of UBA5 in the erythroid lineage rescues the UBA5-deficient embryos from anemia and prolongs their survival, revealing that the UFM1-conjugating system has an essential role in erythroid differentiation. However, it is necessary to clarify whether UBA5 has other functions distinct from protein conjugation in the control of erythrocyte biogenesis in mice, as UBA4, the E1 enzyme of the Urm1 system, is known to function in tRNA uracyl thiolation in yeast, independent of protein modification by Urm1 (18).

\section{UFC1}

UFC1 (also known as HSPC155) is an UFM1-conjugating E2 enzyme consisting of 167 amino acids with a predicted molecular mass of $19.4 \mathrm{kDa}$. Its gene is located in human chromosome 1q23.3. UFC1 is mainly localized in the nucleus and partly in the cytoplasm, but excluded from the nucleoli (http: //www.proteinatlas.org). UFC1 shows low sequence homology (within a range of 13-17\%) with other E2 enzymes (23). However, UFC1 has the catalytic core domain conserved in all E2-like enzymes, except that it contains an additional $\mathrm{N}$-terminal helix. The active site Cys116 is located in a flexible loop that is highly solvent accessible. Upon binding of UFC1 to the ubiquitin-fold domain of UBA5, UFM1 is transferred to the cysteine residue of UFC1 by a transesterification reaction.

The neuronal cell adhesion molecule (NCAM) plays important roles in the control of cell migration, synaptogenesis, and axonal outgrowth (24). Recently, NCAM140, an isoform of NCAM, was shown to interact with UFC1 upon analysis by protein macroarray and ELISA (24). NCAM140 and UFC1 co-localize in the surface of B35 neuroblastoma cells and overexpression of UFM1 increases NCAM140 endocytosis. Therefore, UFM1 has been suggested to play a role in trafficking of cell surface molecules, although it remains unknown whether NCAM140 or other cell surface proteins are modified by UFM1.

\section{UFL1}

UFL1 (also known as Maxer, NLBP, and RCAD) is an UFM1 E3 ligase consisting of 794 amino acids with a predicted molecular mass of $89.5 \mathrm{kDa}$. Its gene is located in human chromosome $6 \mathrm{q} 16.1$. UFL1 has a transmembrane domain and localizes predominantly in ER membrane. It also has a nuclear localization signal (NLS) sequence, which is functional only when the transmembrane domain is deleted $(25,26)$.

UFL1 does not have any domain conserved for ubiquitin E3 ligases, such as HECT, RING finger, and U-box. However, its N-terminal region (amino acid sequence: 1-202) is highly conserved across species, and sufficient for the transfer of UFM1 from UFC1 to C20orf116, the first target substrate identified for ufmylation, under both in vitro and in vivo conditions (26). Since the 
$\mathrm{N}$-terminal region lacks the active site cysteine residue, which is typically found in HECT type E3 ligase for transthiolation reaction, UFL1 may play a role as a scaffold protein that recruits E2 enzyme and target proteins similarly to RING type ubiquitin E3 ligase.

\section{UFBP1}

UFM1-binding protein 1 (UFBP1: also known as Dashurin and DDRGK1) consists of 314 amino acids with a predicted molecular mass of $35.6 \mathrm{kDa}$. Its gene is located in human chromosome $20 \mathrm{q} 13$. UFBP1 contains a transmembrane helix (amino acid sequence: 4-21), a NLS sequence (64-68), a PCI [proteasome, COP9, and initiation factor domain (228-272)], and a DDRGK sequence (253-267) (27). UFBP1 also has an N-terminal signal sequence (126) for its localization in ER. However, deletion of the N-terminal signal sequence leads to nuclear localization of UFBP1.

UFBP1 was originally identified as C20orf116, which is the first target protein identified for ufmylation (26). It interacts not only with UFM1 but also with UFL1 and target proteins for ufmylation, such as ASC1 and LZAP. Depletion of UFBP1 abrogates ufmylation of the target proteins, indicating that UFBP1 serves as a cofactor as well as a substrate for ufmylation. Interestingly, ASC1 ufmylation could also be prevented by substitution of the UFM1 acceptor site Lys267 in UFBP1 with Arg. Moreover, this Lys-to-Arg mutation markedly reduces the interaction of UFBP1 with UFL1, although not with ASC1. Thus, it appears that UFBP1 may first act as a substrate of UFL1 through their weak binding and the ufmylated UFBP1, then binds to the ligase with high affinity, which might be required for the activation of UFL1. Figure 2 summarizes the overall process of protein ufmylation.

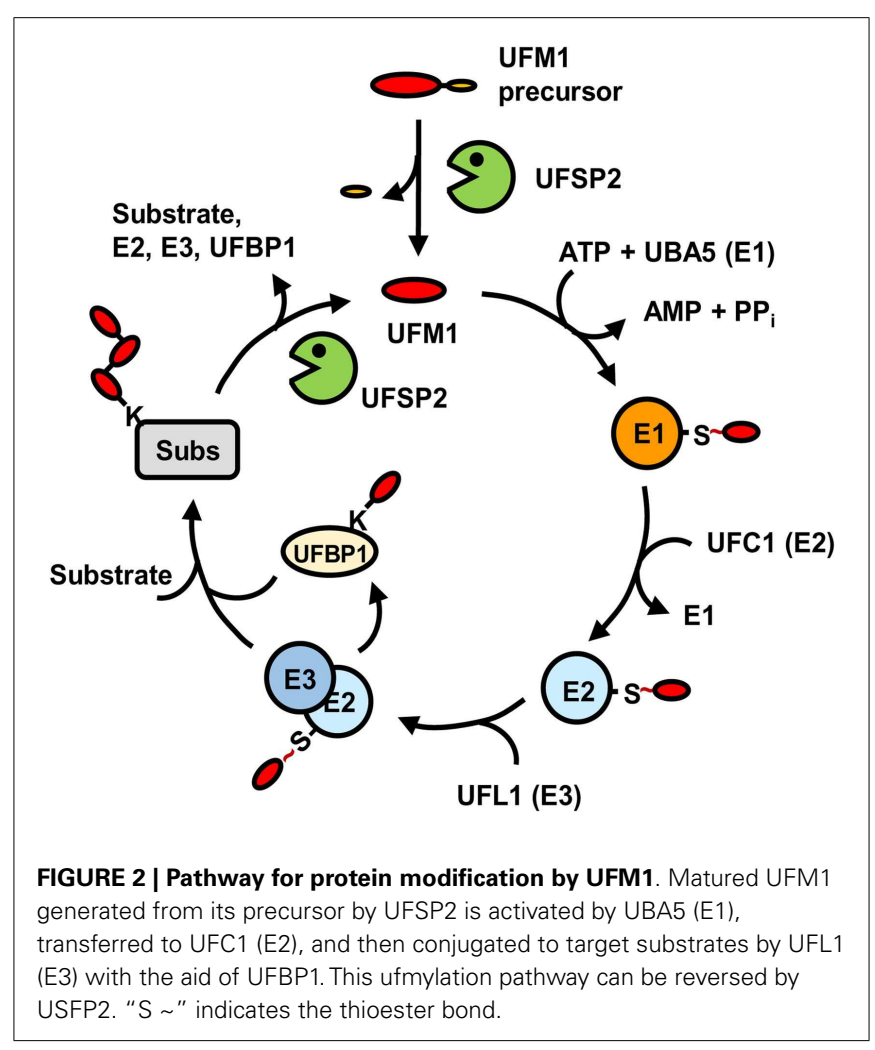

\section{UFM1-SPECIFIC PR0TEASES}

\section{UFSP1}

Mouse Ufsp1 consists of 217 amino acids with a predicted molecular mass of $23.4 \mathrm{kDa}$. Like most deubiquitinating enzymes (DUBs) and ubiquitin-like protein-specific proteases (ULPs), mouse Ufsp1 belongs to the family of cysteine proteases. However, it shows no sequence homology to previously known proteases (28). This novel cysteine protease has a papain-like fold with a unique active site that is composed of a Cys box and a conserved "Asp-ProHis" box, instead of the canonical Cys-His-Asp catalytic triad. This novel active site configuration appears to form a new subfamily of the cysteine protease superfamily (28).

Human USFP1 consists of 142 amino acids with a predicted molecular mass of $15.0 \mathrm{kDa}$. Its gene is located in human chromosome 7q22.1. However, unlike the catalytically active mouse Ufsp1, human UFSP1 is expected to be non-functional, since it is shorter on the N-terminus and thereby lacks the conserved cysteine active site (Figure 3).

\section{UFSP2}

Human USFP2 consists of 469 amino acids with a predicted molecular mass of $53.16 \mathrm{kDa}$. Its gene is located in chromosome 4q35.1. The crystal structure of mouse UFSP2 shows that the protease is composed of two domains (29). The C-terminal catalytic domain is similar to UFSP1 with the active site composed of a Cys box and a conserved Asp-Pro-His box. The novel N-terminal domain shows a unique structure and plays a role in the recognition of UFBP1. UFSP2 resides in both the nucleus and the cytoplasm. However, overexpressed N-terminal domain co-localizes with UFBP1 in ER, where UFBP1 predominantly localizes, suggesting that the N-terminal domain of UFSP2 plays an important role in the recruitment of UFBP1 to ER for reversal of ufmylation process.

A mutation within the human UFSP2 gene has been identified in a family with an autosomal dominant form of hip dysplasia, called Beukes familial hip dysplasia (29). This mutation predicts the replacement of the highly conserved Tyr 290 by His in the encoded protein. Interestingly, the substitution of Tyr282 in mouse UFSP2, which is equivalent to Tyr290 in human UFSP2, abolishes the in vitro UFM1-processing activity (22). Thus, it appears that impairment of reversible modification of unknown protein(s) by UFM1 is associated with an autosomal dominant form of hip dysplasia.

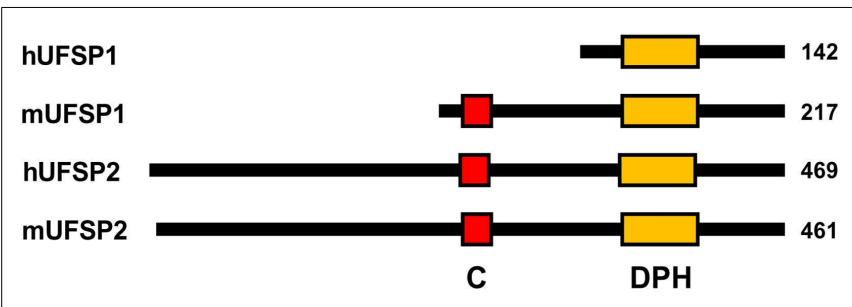

FIGURE 3 | Schematic diagram for primary structures of UFBPs. " $C$ " and "DPH" denote the active site Cys-box (red) and Asp-Pro-His box (orange), respectively. Note that hUFSP1 lacks the Cys box. 


\section{ROLE OF ASC1 UFMYLATION IN BREAST CANCER DEVELOPMENT \\ IDENTIFICATION OF ASC1 AS A TARGET FOR UFMYLATION}

Although UFBP1 (C20orf116) was identified as the first target protein for ufmylation (26), its biological function remained unknown. Recently, however, numerous target proteins for ufmylation have been identified by stable expression of Flag-HisUFM1 in NIH3T3 and double affinity purification using $\mathrm{Ni}^{2+}$ nitrilotriacetic acid-conjugated agarose and anti-Flag antibodyconjugated resins, followed by mass spectrometry (22). The identified ufmylated proteins include ASC1, a transcriptional coactivator of ER $\alpha$, and LZAP (also known as CDK5RAP3 and C53) that has tumor suppressive functions, including activation of p53, induction of apoptosis, and suppression of NF- $\mathrm{\kappa B}$ signaling (30-32).

All of Lys324, Lys325, Lys334, and Lys367 in ASC1 serve as the acceptor sites for UFM1 (22). Of the six lysine residues in UFM1, only Lys69 is involved in poly-UFM1 chain formation on ASC1 via isopeptide bond linkage. However, it is possible that other lysine residues may also participate in poly-UFM1 chain formation on other target proteins. Knockdown of any of UBA5 (E1), UFC1 (E2), UFL1 (E3), and UFBP1 abrogates poly-UFM1 chain formation on ASC1, indicating that UFBP1 serves as an essential cofactor for ufmylation process.

\section{REQUIREMENT OF ESTROGEN FOR ASC1 UFMYLATION}

Endogenous ASC1 can be ufmylated upon treatment of ER $\alpha$ negative cells with estrogen, but not without it (22). In the absence of estrogen, UFSP2 remains bound to the N-terminal zinc-finger domain of ASC1, and rapidly removes UFM1 molecules that are conjugated to ASC1. In the presence of the hormone, ER $\alpha$ forms a dimeric complex, translocates to the nucleus, and displaces UFSP2 for its binding to the zinc-finger domain of ASC1, thus allowing ASC1 ufmylation. On the other hand, no ufmylation of ASC1 can be observed in ER $\alpha$-negative cells, such as MDA-MB-453, regardless of the presence of estrogen. In addition, 4-hydroxy-tamoxifen, an $\mathrm{ER} \alpha$ antagonist, abrogates ASC1 ufmylation by preventing the interaction of $\mathrm{ASC} 1$ with $\mathrm{ER} \alpha$, indicating the requirement of estrogen binding to ER $\alpha$ for ASC1 ufmylation.

ASC1 acts as a general transcriptional co-activator of nuclear hormone receptors upon binding to not only ER $\alpha$ but also other nuclear receptors, such as androgen receptor (AR) (15). Accordingly, dihydrotestosterone (DHT), an AR agonist, induces ASC1 ufmylation in LNCap (AR-positive) cells, but not in PC3 (AR-negative) cells (22). Thus, ligand-dependent ASC1 ufmylation appears specific to cognate nuclear hormone receptors and it is likely that ligands for other nuclear receptors (e.g., all-trans-retinoic acid for RAR $\alpha$ ) can induce ASC1 ufmylation.

\section{REQUIREMENT OF ASC1 UFMYLATION FOR ER $\alpha$ TRANSACTIVATION}

The zinc-finger domain of ASC1 serves as a binding site for nuclear hormone receptors, transcriptional co-activators (e.g., SRC1 and p300), and basic transcriptional machinery (e.g., TFIIA and TBP) (14). Thus, ASC1 plays an important role as a platform that recruits the necessary components for nuclear receptor-mediated transcription. However, it remained unclear how the zinc-finger domain, a short region in ASC1 (amino acid sequence: 125-237), can simultaneously interact with such a group of the proteins. Remarkably, poly-UFM1 chain conjugated to ASC1 plays a crucial role as a scaffold protein that recruits SRC1, p300, ER $\alpha$, and ASC1 itself to estrogen-responsive elements (EREs) located in the promoters of ER $\alpha$ target genes, such as $p S 2$, Cyclin $D$, and $c-M Y C(22,33,34)$. Moreover, this recruitment leads to a dramatic increase in ER $\alpha$ transactivation. Whereas knockdown of UFSP2 markedly promotes ER $\alpha$ transactivation, its overexpression abolishes it. Knockdown of UBA5 or overexpression of an ufmylation-deficient ASC1 mutant, in which the four UFM1 acceptor lysine residues are replaced by arginine, also abrogates $\mathrm{ER} \alpha$ transactivation, indicating that ASC1 ufmylation is crucial for ER $\alpha$ transactivation.

\section{PROMOTION OF TUMOR FORMATION BY ASC1 UFMYLATION}

Recently, colony-forming assay has shown that estrogen-induced ASC1 ufmylation is critically involved in anchorage-independent growth of ER $\alpha$-positive MCF7 breast cancer cells (22). Xenograft analysis using ovariectomized mice has further revealed that ASC1 ufmylation is tightly associated with estrogen-dependent tumor formation in vivo (22). Whereas depletion of ASC1, UBA5, or both prevent colony formation and tumor growth, overexpression of ASC1 markedly increases them. Remarkably, depletion of UFSP2 most dramatically promotes the cell growth and tumor formation, and this promotion can be abrogated by simultaneous depletion of ASC1, implicating the role of UFSP2 as a tumor suppressor. In addition, tamoxifen could completely reverse the stimulatory effects of ASC1 overexpression and UFSP2 depletion on colony formation and tumor growth. These findings implicate a crucial role of ASC1 ufmylation in development of ER $\alpha$ positive breast cancer by promoting ER $\alpha$ transactivity. Figure 4 summarizes estrogen-induced ASC1 ufmylation pathway for ER $\alpha$ transactivation, which leads to development of breast cancer.

\section{POSSIBILITY FOR DEVELOPMENT OF ANTI-BREAST CANCER DRUG}

Breast cancer is one of the most prevailing cancers of woman. It is well-known that estrogen plays a critical role in the pathogenesis and development of breast cancer (35). Moreover, nearly $70 \%$ of breast cancer is $E R \alpha$-positive (36). Therefore, patients with $\mathrm{ER} \alpha$-positive cancer have been treated with aromatase inhibitors, which prevent the synthesis of estrogen or with tamoxifen, which blocks the binding of estrogen to ER $\alpha$ (35-38). These treatments are highly effective, but many patients inevitably develop the drug-resistant invasive tumors. Therefore, new drugs against $\mathrm{ER} \alpha$-positive breast cancer are of high demand.

As to the findings that estrogen-induced ASC1 ufmylation is required for ER $\alpha$ transactivation and tumor formation (22), UBA5 and other components of UFM1-conjugating machinery involved in ASC1 ufmylation could be used as potential targets for development of new therapeutic drugs against $\mathrm{ER} \alpha$-positive breast cancer. Significantly, the induction of ASC1 ufmylation is not limited to estrogen, but could also occur in the presence of other ligands, such as testosterone and retinoic acid, if their cognate nuclear receptors are present in cells (22). Thus, the components of UFM1conjugating system may also represent potent therapeutic targets in patients with other nuclear receptor-related cancers, such as prostate and leukemic cancers. Since UFSP2 knockdown leads 


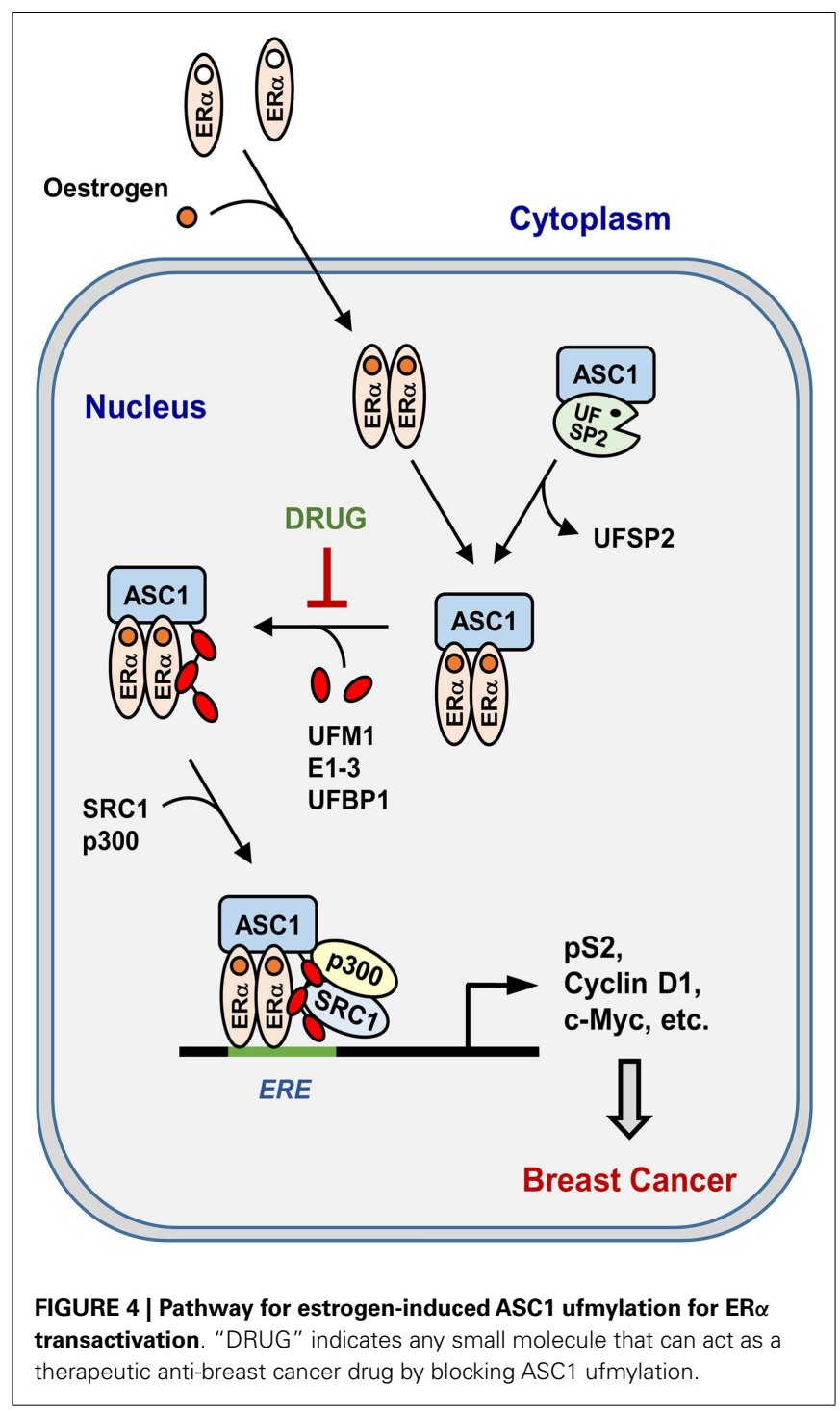

to the most dramatic effect on the increase in cell proliferation, anchorage-independent colony formation, and tumor formation, small molecules that increase the affinity of UFSP2 to the zincfinger domain of ASC1, or the activity of the protease could also be used as a potential drug against the nuclear receptor-mediated cancers.

\section{OTHER BIOLOGICAL FUNCTIONS OF THE UFM1-CONJUGATING SYSTEM \\ UFL1 IN TUMORIGENESIS}

The human UFL1 gene is located in chromosome 6q16.1, a region that was reported to be frequently lost in prostate and gastric cancers as well as in bile duct cancer cell lines (39-41). It has also been reported that the expression of UFL1 (also called NLBP, RCAD, and Maxer) cannot be detected in invasive hepatocellular carcinoma cells including HepG2, Hep3B, HLE, and PLC, whereas it can be detected in non-invasive Huh7 hepatocellular carcinoma cell line (39). In addition, UFL1 was shown to cooperate with LZAP in suppression of cell invasion and NF- $\kappa \mathrm{B}$ signaling by mutual stabilization, suggesting that UFL1 may act as a tumor suppressor (27).

However, it has also been reported that UFL1 knockdown suppresses the proliferation of C6 glioma cells and LZAP-mediated inhibition of Cyclin D1 transcription (25). In addition, UFL1 is highly expressed in human lung adenocarcinoma and its overexpression promotes the proliferation of rat H1299 lung cancer cells through interaction with p120 catenin, suggesting that UFL1 may play a role in development of lung carcinoma (42). Thus, UFL1 seems to have two opposite functions: one in tumor suppression and the other in tumor development, perhaps depending on its target proteins for ufmylation in different types of cells and tissues. In this respect, it would be of high interest to see if UFL1-mediated ufmylation differentially influences the function of LZAP and p120 catenin in the control of tumorigenesis, although it is also possible that UFL1 may regulate their functions independently of its E3 ligase activity.

Interestingly, UFBP1 was shown to bind to I- $\kappa \mathrm{B}$, stabilize it, and thereby inhibit NF- $\kappa$ B signaling (43). In addition, UFBP1 knockdown leads to inhibition of cell migration and invasion. Thus, UFBP1, like UFL1, plays two opposite roles as a tumor suppressor by inhibiting NF- $\mathrm{B}$ signaling and as a tumor promoter by serving as a cofactor of the UFM1-conjugating system for ASC1 in development of breast cancer. UFBP1 may regulate NF- $\kappa$ B pathway independently of its cofactor function in ufmylation. Further studies are required to clarify the opposite dual functions of UFBP1 and UFL1 in the control of tumorigenesis and NF- $\kappa$ B signaling.

\section{THE UFM1 SYSTEM IN ER STRESS RESPONSE}

The expression of UFM1 was shown to be up-regulated in type 2 diabetes and ischemic heart disease in mice, whose pathological conditions are associated with activation of ER stress response (44-47). ER stress induced by cyclopiazonic acid or thapsigargin, both of which are inhibitors of the ER $\mathrm{Ca}^{2+}$ ATPase pump, was also shown to increase the expression of UFM1, UFBP1, and UFL1 (48). Interestingly, this increase attenuates ER stress-induced apoptosis of mouse pancreatic $\beta$-cells. Recently, brefeldin, an inhibitor of vesicle trafficking, has been shown to increase the transcript level of UFM1, UFBP1, and UFL1, and this increase could not be observed in $\mathrm{Xbp1}^{-/-}$MEFs (45). These findings suggest that the UFM1-conjugating system plays an important role in maintaining the ER homeostasis.

\section{CONCLUDING REMARKS}

The UFM1-conjugating machinery, consisting of UBA5 (E1), UFC1 (E2), UFL1 (E3), and UFBP1, is the most recently discovered post-translational protein modification system, whose biological function is largely unknown. Intriguingly, estrogen-induced ASC1 ufmylation by this system plays a crucial role in development of breast cancer by promoting ER $\alpha$-transactivation. Thus, each component of the UFM1-conjugating machinery and UFSP2 that reverses ufmylation process could be potential targets for development of drug against ER $\alpha$-positive breast cancer. Since ASC1 ufmylation can be achieved by specific ligands for other nuclear receptors, such as AR and RAR $\alpha$, it would be of interest to examine whether ASC1 ufmylation also promotes the transcriptional activity of the nuclear receptors and thereby the receptor-mediated 
cancers, such as prostate and leukemic cancers, respectively. Up to present, however, the knowledge on biological function of protein ufmylation is limited to ASC1. Significantly, ER stress induces the expression of UBA5, UFBP1, and UFL1, suggesting that proteins involved in UPR response may be potential targets for ufmylation. Thus, extensive studies are of necessity to identify more target proteins for ufmylation and explore their biological function.

\section{ACKNOWLEDGMENTS}

This work was supported by the grants from National Research Foundation of Korea (NRF-2005-084-C00025) and KOSEF (M1053301001-05N3301), and from The Institute for Protein Metabolism. HY was the recipient of the BK21 fellowship. We apologize for the event that any relevant publications were inadvertently omitted.

\section{REFERENCES}

1. Jeon YJ, Yoo HM, Chung CH. ISG15 and immune diseases. Biochim Biophys Acta (2010) 1802:485-96. doi:10.1016/j.bbadis.2010.02.006

2. Flotho A, Melchior F. Sumoylation: a regulatory protein modification in health and disease. Annu Rev Biochem (2013) 82:357-85. doi:10.1146/annurevbiochem-061909-093311

3. Geoffroy M-C, Hay RT. An additional role for SUMO in ubiquitin-mediated proteolysis. Nat Rev Mol Cell Biol (2009) 10:564-8. doi:10.1038/nrm2707

4. Hoeller D, Hecker C-M, Dikic I. Ubiquitin and ubiquitin-like proteins in cancer pathogenesis. Nat Rev Cancer (2006) 6:776-88. doi:10.1038/nrc1994

5. Komatsu M, Chiba T, Tatsumi K, Iemura S, Tanida I, Okazaki N, et al. A novel protein-conjugating system for Ufm 1, a ubiquitin-fold modifier. EMBOJ (2004) 23:1977-86. doi:10.1038/sj.emboj.7600205

6. Daniel J, Liebau E. The ufm1 cascade. Cells (2014) 3:627-38. doi:10.3390/ cells3020627

7. Zhou W, Slingerland JM. Links between oestrogen receptor activation and proteolysis: relevance to hormone-regulated cancer therapy. Nat Rev Cancer (2014) 14:26-38. doi:10.1038/nrc3622

8. Shao W, Keeton EK, McDonnell DP, Brown M. Coactivator AIB1 links estrogen receptor transcriptional activity and stability. Proc Natl Acad Sci U S A (2004) 101:11599-604. doi:10.1073/pnas.0402997101

9. Xu J, Wu R-C, O'Malley BW. Normal and cancer-related functions of the p160 steroid receptor co-activator (SRC) family. Nat Rev Cancer (2009) 9:615-30. doi: $10.1038 /$ nrc2695

10. Hassig A, Schreiberi SL. Nuclear histone acetylases and deacetylases regulation: HATS off to HDACs. Curr Opin Chem Biol (1997) 1:300-8. doi:10.1016/S13675931(97)80066-X

11. Lee DY, Teyssier C, Strahl BD, Stallcup MR. Role of protein methylation in regulation of transcription. Endocr Rev (2005) 26:147-70. doi:10.1210/er.2004-0008

12. Lonard DM, O'malley BW. Nuclear receptor coregulators: judges, juries, and executioners of cellular regulation. Mol Cell (2007) 27:691-700. doi:10.1016/j. molcel.2007.08.012

13. Lee JW, Choi HS, Gyuris J, Brent R, Moore DD. Two classes of proteins dependent on either the presence or absence of thyroid hormone for interaction with the thyroid hormone receptor. Mol Endocrinol (1995) 9:243-54. doi:10.1210/mend.9.2.7776974

14. Kim HJ, Yi JY, Sung HS, Moore DD, Jhun BH, Lee YC, et al. Activating signal cointegrator 1, a novel transcription coactivator of nuclear receptors, and its cytosolic localization under conditions of serum deprivation. Mol Cell Biol (1999) 19:6323-32.

15. Lee YS. Activating signal cointegrator 1 is highly expressed in murine testicular Leydig cells and enhances the ligand-dependent transactivation of androgen receptor. Biol Reprod (2002) 67:1580-7. doi:10.1095/biolreprod.102.006155

16. Jung D, Sung H, Goo Y, Lee HM, Park OK, Jung S, et al. Novel transcription coactivator complex containing activating signal cointegrator 1. Mol Cell Biol (2002) 22:5203-11. doi:10.1128/MCB.22.14.5203-5211.2002

17. Sasakawa H, Sakata E, Yamaguchi Y, Komatsu M, Tatsumi K, Kominami E, et al. Solution structure and dynamics of Ufm 1, a ubiquitin-fold modifier 1. Biochem Biophys Res Commun (2006) 343:21-6. doi:10.1016/j.bbrc.2006.02.107
18. Schulman BA, Harper JW. Ubiquitin-like protein activation by E1 enzymes: the apex for downstream signalling pathways. Nat Rev Mol Cell Biol (2009) 10:319-31. doi:10.1038/nrm2673

19. Bacik J-P, Walker JR, Ali M, Schimmer AD, Dhe-Paganon S. Crystal structure of the human ubiquitin-activating enzyme 5 (UBA5) bound to ATP: mechanistic insights into a minimalistic E1 enzyme. J Biol Chem (2010) 285:20273-80. doi:10.1074/jbc.M110.102921

20. Zheng M, Gu X, Zheng D, Yang Z, Li F, Zhao J, et al. UBE1DC1, an ubiquitinactivating enzyme, activates two different ubiquitin-like proteins. J Cell Biochem (2008) 104:2324-34. doi:10.1002/jcb.21791

21. Tatsumi K, Yamamoto-Mukai H, Shimizu R, Waguri S, Sou Y-S, Sakamoto A, et al. The Ufml-activating enzyme Uba5 is indispensable for erythroid differentiation in mice. Nat Commun (2011) 2:181. doi:10.1038/ncomms1182

22. Yoo HM, Kang SH, Kim JY, Lee JE, Seong MW, Lee SW, et al. Modification of ASC1 by UFM1 is crucial for $\mathrm{ER} \alpha$ transactivation and breast cancer development. Mol Cell (2014) 56:261-74. doi:10.1016/j.molcel.2014.08.007

23. Liu G, Forouhar F, Eletsky A, Atreya HS, Aramini JM, Xiao R, et al. NMR and XRAY structures of human E2-like ubiquitin-fold modifier conjugating enzyme 1 (UFC1) reveal structural and functional conservation in the metazoan UFM1UBA5-UFC1 ubiquination pathway. J Struct Funct Genomics (2009) 10:127-36. doi:10.1007/s10969-008-9054-7

24. Homrich M, Wobst H, Laurini C, Sabrowski J, Schmitz B, Diestel S. Cytoplasmic domain of NCAM140 interacts with ubiquitin-fold modifierconjugating enzyme-1 (Ufc1). Exp Cell Res (2014) 324:192-9. doi:10.1016/j. yexcr.2014.04.003

25. Shiwaku H, Yoshimura N, Tamura T, Sone M, Ogishima S, Watase K, et al. Suppression of the novel ER protein Maxer by mutant ataxin-1 in Bergman glia contributes to non-cell-autonomous toxicity. EMBO J (2010) 29:2446-60. doi:10.1038/emboj.2010.116

26. Tatsumi K, Sou Y, Tada N, Nakamura E, Iemura S, Natsume T, et al. A novel type of E3 ligase for the Ufm 1 conjugation system. J Biol Chem (2010) 285:5417-27. doi:10.1074/jbc.M109.036814

27. Wu J, Lei G, Mei M, Tang Y, Li H. A novel C53/LZAP-interacting protein regulates stability of C53/LZAP and DDRGK domain-containing protein 1 (DDRGK1) and modulates NF-kappaB signaling. J Biol Chem (2010) 285:15126-36. doi:10.1074/jbc.M110.110619

28. Ha BH, Ahn H-C, Kang SH, Tanaka K, Chung CH, Kim EEK. Structural basis for Ufml processing by UfSP1. J Biol Chem (2008) 283:14893-900. doi:10.1074/jbc.M708756200

29. Ha BH, Jeon YJ, Shin SC, Tatsumi K, Komatsu M, Tanaka K, et al. Structure of ubiquitin-fold modifier 1-specific protease UfSP2. J Biol Chem (2011) 286:10248-57. doi:10.1074/jbc.M110.172171

30. Jiang H, Luo S, Li H. Cdk5 activator-binding protein $\mathrm{C} 53$ regulates apoptosis induced by genotoxic stress via modulating the G2/M DNA damage checkpoint. J Biol Chem (2005) 280:20651-9. doi:10.1074/jbc.M413431200

31. Wang J, An H, Mayo MW, Baldwin AS, Yarbrough WG. LZAP, a putative tumor suppressor, selectively inhibits NF-kappaB. Cancer Cell (2007) 12:239-51. doi:10.1016/j.ccr.2007.07.002

32. Wang J, He X, Luo Y, Yarbrough WG. A novel ARF-binding protein (LZAP) alters ARF regulation of HDM2. Biochem J (2006) 393:489-501. doi:10.1042/ BJ20050960

33. Nilsson S, Mäkelä S, Treuter E, Tujague M, Thomsen J, Andersson G, et al. Mechanisms of estrogen action. Physiol Rev (2001) 81:1535-65.

34. Klinge CM. Estrogen receptor interaction with estrogen response elements. Nucleic Acids Res (2001) 29:2905-19. doi:10.1093/nar/29.14.2905

35. Umar A, Dunn BK, Greenwald P. Future directions in cancer prevention. Nat Rev Cancer (2012) 12:835-48. doi:10.1038/nrc3397

36. Jozwik KM, Carroll JS. Pioneer factors in hormone-dependent cancers. Nat Rev Cancer (2012) 12:381-5. doi:10.1038/nrc3263

37. Beelen K, Zwart W, Linn SC. Can predictive biomarkers in breast cancer guide adjuvant endocrine therapy? Nat Rev Clin Oncol (2012) 9:529-41. doi:10.1038/nrclinonc.2012.121

38. Nilsson S, Koehler KF, Gustafsson J-Å. Development of subtype-selective oestrogen receptor-based therapeutics. Nat Rev Drug Discov (2011) 10:778-92. doi:10.1038/nrd3551

39. Li BC, Chan WY, Li CY, Chow C, Ng EK, Chung SC. Allelic loss of chromosome 6q in gastric carcinoma. Diagn Mol Pathol (2003) 12:193-200. doi:10.1097/00019606-200312000-00003 
40. Lu T, Hano H. Deletion at chromosome arms 6q16-22 and 10q22.3-23.1 associated with initiation of prostate cancer. Prostate Cancer Prostatic Dis (2008) 11:357-61. doi:10.1038/pcan.2008.4

41. Saito S, Ghosh M, Morita K, Hirano T. The genetic differences between gallbladder and bile duct cancer cell lines. Oncol Rep (2006) 16:949-56. doi:10.3892/or.16.5.949

42. Kim CH, Nam H-S, Lee EH, Han SH, Cho HJ, Chung HJ, et al. Overexpression of a novel regulator of p120 catenin, NLBP, promotes lung adenocarcinoma proliferation. Cell Cycle (2013) 12:2443-53. doi:10.4161/cc.25451

43. Xi P, Ding D, Zhou J, Wang M, Cong Y-S. DDRGK1 regulates NF-кB activity by modulating IкB $\alpha$ stability. PLoS One (2013) 8:e64231. doi:10.1371/journal. pone.0064231

44. Lee Y, Johnson KR, Hallenbeck JM. Global protein conjugation by ubiquitinlike-modifiers during ischemic stress is regulated by microRNAs and confers robust tolerance to ischemia. PLoS One (2012) 7:e47787. doi:10.1371/journal. pone. 0047787

45. Zhang Y, Zhang M, Wu J, Lei G, Li H. Transcriptional regulation of the Ufm1 conjugation system in response to disturbance of the endoplasmic reticulum homeostasis and inhibition of vesicle trafficking. PLoS One (2012) 7:e48587. doi:10.1371/journal.pone.0048587

46. Lu H, Yang Y, Allister EM, Wijesekara N, Wheeler MB. The identification of potential factors associated with the development of type 2 diabetes: a quantitative proteomics approach. Mol Cell Proteomics (2008) 7:1434-51. doi:10.1074/mcp.M700478-MCP200

47. Azfer A, Niu J, Rogers LM, Adamski FM, Kolattukudy PE. Activation of endoplasmic reticulum stress response during the development of ischemic heart disease. Am J Physiol Heart Circ Physiol (2006) 291:H1411-20. doi:10.1152/ ajpheart.01378.2005

48. Lemaire K, Moura RF, Granvik M, Igoillo-Esteve M, Hohmeier HE, Hendrickx $\mathrm{N}$, et al. Ubiquitin fold modifier 1 (UFM1) and its target UFBP1 protect pancreatic beta cells from ER stress-induced apoptosis. PLoS One (2011) 6:e18517. doi:10.1371/journal.pone.0018517

Conflict of Interest Statement: The authors declare that the research was conducted in the absence of any commercial or financial relationships that could be construed as a potential conflict of interest.

Received: 21 February 2015; accepted: 03 March 2015; published online: 20 March 2015.

Citation: Yoo HM, Park JH, Jeon YJ and Chung CH (2015) Ubiquitin-fold modifier 1 acts as a positive regulator of breast cancer. Front. Endocrinol. 6:36. doi: $10.3389 /$ fendo.2015.00036

This article was submitted to Cancer Endocrinology, a section of the journal Frontiers in Endocrinology.

Copyright $@ 2015$ Yoo, Park, Jeon and Chung. This is an open-access article distributed under the terms of the Creative Commons Attribution License (CC BY). The use, distribution or reproduction in other forums is permitted, provided the original author(s) or licensor are credited and that the original publication in this journal is cited, in accordance with accepted academic practice. No use, distribution or reproduction is permitted which does not comply with these terms. 